\title{
GRUPOS NA ENFERMAGEM: CLASSIFICAÇÃO, TERMINOLOGIAS E FORMAS DE ABORDAGEM ${ }^{a}$
}

\author{
Workgroups in nursing: classification, terminologies and approaches ${ }^{a}$ \\ Grupos en enfermería: classificación, terminología y formas de enfoque ${ }^{a}$
}

Fabiana Verdan Simões

Marluci Andrade Conceição Stipp

\begin{abstract}
Resumo
Este artigo retrata as diversas terminologias utilizadas para os trabalhos de grupos realizados por enfermeiros e contextualiza as formas de abordagens utilizadas em estudos de enfermeiros sobre as atividades grupais. Aborda as seguintes terminologias: grupos operativos e seus campos de atuação, grupos com pacientes somáticos, grupos interdisciplinares homogêneos, grupos de convivência, grupos focais e grupos de convergência. As terminologias existentes são pautadas de acordo com objetivos mais definidos de atuação do grupo, onde, na verdade, todas se encaixam no conceito de grupo operativo, como uma classificação dessas atividades grupais. Sobre as formas de abordagem relacionadas a essas atividades, são descritas: grupos como processo educativo em saúde, grupos como tecnologia e como estratégia. Entre as citadas, a visão do grupo como estratégia, segundo os autores, permite ampliar o significado de atuação do trabalho de grupo e sua importância como ferramenta de trabalho para o profissional enfermeiro.
\end{abstract}

Palavras-chave: Educação em Enfermagem. Terminologia. Classificação. Educação do paciente.

\begin{abstract}
This study defines different terminologies used in group work carried out by nurses and contextualises lines of approaches used in nurse's studies about group activities. If approaches the following terminologies: Operatives groups and their respective action areas, group of somatic patients, homogeneous interdisciplinary groups, interactive groups, focal groups and convergence groups. The objectives of the existing terminologies are focused on their objectives, where in reality all terminologies fit in the concept of operative groups as a classification of such. The following approaches related to those activities are described as follows: Health Education Groups and Technology and Strategy Groups. Among the ones cited above, the focus on the strategy groups, according to their authors, allows for a better group performance and its importance as a work tool for the professional nurse.
\end{abstract}

\section{Keywords:}

Education, Nursing. Terminology. Classification.

Patient Education.

\section{Resumen}

Este artículo trata de las variadas terminologías utilizadas para los trabajos de grupos realizados por enfermeros y contextualiza las formas de enfoques empleadas en estudos de enfermeros sobre las actividades grupales. Enfoca las siguientes terminologías: grupos operativos y sus campos de actuación, grupos con pacientes somáticos, grupos interdisciplinarios homogéneos, grupos de convivir, grupos focales y grupos de convergencia. Las terminologías que hay son pautadas de acuerdo con objetivos más definidos de actuación del grupo, donde, en verdad, todas se encajan en el concepto de grupo operativo, como una clasificación de actividades grupales. Sobre las formas de abordaje que tienen relación con esas actividades, son descriptas: grupos como proceso educativo en salud, grupos como tecnología y como estrategía. A lo mejor, la visión del grupo como estrategía, de acuerdo con los autores, puede crecer su significado de actuación del trabajo de grupo y su importancia como herramienta de trabajo para el profesional enfermero.

Palabras clave:

Educación en Enfermería. Terminología. Classificación.

Educación del Pacientce 


\section{INTRODUÇÃO}

A ampla gama de aplicações das atividades grupais e seus benefícios mostram a necessidade e importância deste tema. Na Enfermagem, esta prática é muito utilizada na Saúde Pública e no âmbito hospitalar em ambulatórios, sendo atualmente difundida também na assistência de enfermagem com clientela internada nas diferentes especialidades.

Para contextualizar esta temática, precisamos conhecer a acepção da palavra grupo, que segundo a visão de Pichon-Riviéril:169, é um conjunto de pessoas interagindo em tempo e espaço articuladas por sua mútua representação interna, propõe-se explícita ou implicitamente a uma tarefa, que constitui sua finalidade.

A imensa variedade de grupos quanto à estrutura, método, teoria, tipo de participante denota também a sua conjugação, na qual um grupo faz parte do outro ou tem características semelhantes em algum aspecto.

$\mathrm{Na}$ enfermagem, as finalidades desta atividade grupal junto à clientela podem ser educativas ou de informação, reflexão e suporte. A finalidade também pode ser psicoterápica, caso o enfermeiro tenha formação apropriada para a execução das técnicas que levem ao alcance deste objetivo. Mas elas são pouco difundidas na profissão e, quando são utilizadas, desenvolvem-se junto à clientela psiquiátrica.

Quando se busca aprofundamento nas atividades grupais, é condição sine qua non conhecer a Psicossomática para entender melhor os processos grupais.

\section{GRUPOS NA VISÃO DA PSICOSSOMÁTICA}

A expressão Psicossomática, criada por Heinroth, surgiu em 1918. Mas, como movimento, somente consolidou-se em meados do século passado. Este movimento pode ser considerado como uma ideologia sobre saúde e sobre as práticas de saúde, sendo também um campo de pesquisas sobre estes aspectos. A evolução da Psicossomática ocorreu em três fases ${ }^{2: 19}$ :

a) Fase inicial ou psicanalítica: com predomínio dos estudos sobre a gênese inconsciente das enfermidades, sobre as teorias da regressão e sobre os benefícios secundários do adoecer, entre outras;

b) Fase intermediária ou behaviorista: caracterizada pelo estímulo à pesquisa em homens e animais, ao tentar enquadrar os achados à luz das ciências exatas e dar um grande estímulo aos estudos sobre estresse; c) Fase atual ou multidisciplinar: na qual vem emergindo a importância social da visão Psicossomática como uma atividade essencialmente de interação, de interconexão entre vários profissionais de saúde.

Nas atividades da Psicossomática, ou a também denominada Psicologia Médica (visão Psicossomática da Medicina), em instituições de saúde, incluem-se a questão das doenças crônicas e agudas, 0 papel das reações adaptativas ao adoecer, a invalidez, a morte e os recursos terapêuticos extraordinários².

Uma das formas de intervenção da prática psicossomática é a atividade grupal. Adentrando por este campo da Psicossomática, percebe-se uma grande variedade de grupos. Segundo Zimerman ${ }^{3}$, não há um protocolo de classificação geral dos grupos. Mas alguns estudiosos deste campo propõe-se a dividi-los em dois grandes grupos: psicoterápicos e operativos.

Os grupos psicoterápicos envolvem interpretação dos sentimentos e ansiedades da clientela visando a mudança de comportamento, a aquisição de insight, ou seja, uma compreensão repentina, em geral intuitiva, de suas próprias atitudes e comportamentos, de um problema, de uma situação ${ }^{4: 1117}$. Pode-se utilizar diversas técnicas ou teorias das escolas de psicoterapia de grupo, sendo necessário um terapeuta profissional para exercer essa atividade. 0 grupo apresenta uma finalidade terapêutica, que tem um significado não apenas de aliviar ou curar os doentes, mas da própria utilização da psicoterapia.

Os grupos operativos foram introduzidos por Henrique Pichon-Rivièri, na década de 40, na Argentina. 0 grupo era considerado como instrumento de trabalho, um método que cumpria uma função terapêutica ${ }^{5}$.

Atualmente, este conceito de grupos operativos apresenta uma maior abrangência ultrapassando a idéia de método e se fortalecendo na idéia de operatividade englobando vários tipos de grupo, na classificação geral dos grupos. 0 grupo operativo é, genericamente, um continente de todos os demais grupos, inclusive os especificamente psicanalíticos ${ }^{3}$. Essas múltiplas e distintas ramificações de grupos operativos, na prática, não são perfeitamente delimitadas; antes, elas muitas vezes se interpõem, complementam-se e se confundem ${ }^{3: 77-78}$.

Como os grupos operativos englobam até os grupos psicoterápicos ou terapêuticos. Esta terminologia, na visão da psicologia médica, permite respaldar os grupos trabalhados pela enfermagem, como grupos não-terapêuticos. Por isso, a necessidade de uma clas- 
sificação geral de grupos mais definida, tais como grupos psicoterápicos ou terapêuticos e grupos não psicoterápicos ou não-terapêuticos, para melhor compreensão dos vários profissionais não-médicos que pretendem ou se utilizam dessa prática.

É importante salientar que os grupos trabalhados por enfermeiros são não-terapêuticos por não utilizar a psicoterapia na visão da psicologia médica. Mas, entendendo-se terapêutica pela origem da palavra grega therapeía, therapeúein, que significa 'cuidar', 'servir', 'assistir', este termo pode ser empregado pelos benefícios estabelecidos através da diminuição de ansiedades alcançada 4 .

Pichon-Riviéri era psiquiatra, psicanalista considerado um analista singular, único, que transcendia qualquer escola ou grupo, com um grande cabedal de conhecimentos no campo da psicanálise ${ }^{2}$. Combinando psicanálise e psicologia social, construiu uma teoria (quase) completa e complexa, a do grupo operativo.

0 grupo operativo, para Saidon ${ }^{6}$, caracteriza-se por estar centrado, de forma explícita, em uma tarefa que pode ser 0 aprendizado, a cura, o diagnóstico de dificuldades, entre outras. Ao falar de tarefa, Pichon-Rivièri ${ }^{1}$ complementa dizendo que a mesma dependerá do campo operativo do grupo. Assim, num grupo terapêutico, ou seja, psicoterápico, a tarefa é resolver o denominador comum da ansiedade grupal, que adquire características particulares em cada membro. Ele ainda destaca que, quando se trata de um grupo de aprendizagem de psiquiatria ou psicologia clínica2:a7, a tarefa consiste na resolução das ansiedades ligadas à aprendizagem dessas disciplinas, facilitando-se, assim, a assimilação de uma informação realmente operativa.

0 grupo operativo, como continente de todos os demais grupos, é dividido em quatro campos propriamente ditos $3: 76 \cdot 77$. São eles:

-Ensino-aprendizagem: o essencial é aprender a aprender, que 'mais importante do que encher a cabeça de conhecimentos é formar cabeças'. Especificamente em relação à tarefa de aprendizagem e treinamento, são conhecidos os grupos $T$ (Training-groups).

- Institucionais: se destinam a aumentar o rendimento de produção de uma empresa, investindo no pessoal da mesma.

- Comunitários: o melhor exemplo deste tipo de grupo é o de sua crescente aplicação em programas voltados para à saúde mental.
- Terapêuticos: visam fundamentalmente a uma melhoria de alguma situação de patologia dos indivíduos, quer no psiquismo, ou em ambos ao mesmo tempo.

0 grupo de reflexão é considerado um grupo operativo aplicado ao ensino / aprendizagem, descrito por Coronel $^{7}$, que busca a remoção das dificuldades que estão impedindo que o grupo realize sua tarefa. Durante o curso de graduação, em estágio junto à clientela psiquiátrica, uma das pesquisadoras teve a experiência de participar de um grupo de reflexão coordenado por uma enfermeira, cujo objetivo era levar os alunos a desbloquear os sentimentos que pudessem estar dificultando a interação dos alunos com o cliente psiquiátrico, de modo a evitar que os mesmos pudessem desenvolver frustrações pessoais e profissionais.

Atualmente, como enfermeira, ela participa de um grupo de reflexão num grupo chamado "Grupo de Família", no qual participam responsáveis (acompanhantes) de crianças internadas num Hospital de referência em câncer. Nesse grupo, busca-se fornecer um espaço para interação com a equipe de saúde e entre os próprios acompanhantes, esclarecer e dúvidas ou questões pertinentes tornando-se muitas vezes um espaço de "desabafo", com o objetivo de dar suporte para conscientização do papel do acompanhante junto à criança hospitalizada.

Este aprender a aprender dos grupos aplicados ao ensino-aprendizagem também significa aprender a conhecer, um dos quatro pilares da educação. Nele pretende-se conhecer o mundo que nos rodeia, para viver dignamente desenvolvendo capacidades profissionais e de comunicação. Sua finalidade é ter o prazer de compreender, de descobrir8.

No campo institucional, os grupos são realizados com pais, mestres e alunos, em instituições educativas como em escolas; em instituições religiosas como igrejas, com a finalidade de estudo ou caritativa; em instituições laborativas destinando-se a aumentar o rendimento de produção ou investindo em recursos humanos ${ }^{3}$.

Os grupos de auto-ajuda ou auto-sugestão fazem parte dos grupos operativos terapêuticos que têm nos Alcoólicos Anônimos seu exemplo mais conhecido entre outros da mesma natureza. Barros ${ }^{9}$ refere que os grupos de auto-ajuda procuram auxiliar as pessoas a resolver seus problemas relacionados a eventos traumáticos decorrentes de acometimentos de natureza aguda e, em especial, crônica; transtornos aditivos; incapacitações, situações de causas existenciais e traumas. 
Nesse sentido, Zimerman ${ }^{3}$ complementa citando os tipos de participantes através de categorias de prejuízos ou necessidades: Adictos (tabagistas, obesos, drogadictos, alcóolicos, entre outros), cuidados primários de saúde (programas preventivos de saúde, como um suporte para pacientes hipertensos, diabéticos, reumáticos, entre outros), reabilitação (infartados, colostomizados, espancados, mutilados, entre outros), sobrevivência social (estigmatizados, como os homossexuais, os portadores de defeitos físicos, entre outros), suporte (pacientes crônicos físicos ou psíquicos, pacientes terminais, entre outros), problemas sexuais e conjugais (mais utilizados nos Estados Unidos).

Outros tipos de grupos com variadas terminologias encaixam-se dentro dos grupos operativos terapêuticos. Segundo Zimerman3:25, a partir das postulações de Pichon-Rivièri, abriu-se um vasto leque de aplicações dos grupos operativos, as quais, com algumas variações técnicas, são conhecidas por múltiplas e diferentes denominações..

\section{OUTRAS TERMINOLOGIAS DE GRUPOS}

Todos os grupos operativos terapêuticos trabalham com pacientes somáticos. Para Melo Filho ${ }^{10: 188}$, este é o paciente que descreve ou apresenta uma alteração de sua estrutura anatômica ou funções fisiológicas. Por esse motivo, este tipo de grupo também é conhecido como grupo com pacientes somáticos. 0 mesmo autor ${ }^{11: 134}$, ao se referir aos grupos com pacientes somáticos, relata que:

Só a vantagem em reunir pessoas com uma patologia comum, pois percebem melhor seus problemas quando os vêem nos outros (a obesidade, por exemplo); aprendem a tolerar o que repudiam em si. Passam a admitir uma melhor resolução da doença, também pelo que aprenderam com os demais.

Chazan 12:165 usa a terminologia de grupos homogêneos interdisciplinares. Estes são grupos compostos por portadores da mesma patologia, o que permite oferecer-Ihes possibilidades terapêuticas e preventivas que as consultas individuais não atingem ou atingem com menor eficácia. Os objetivos desses grupos são: promover maior adesão ao tratamento; ampliar a consciência sobre sua patologia; desenvolver maior capacidade de assimilação das informações; estimular a solidariedade e favorecer que cada cliente possa atuar, na comunidade, de maneira informal, como um agente de saúde ${ }^{12}$.
Um outro tipo de terminologia é muito utilizada na Enfermagem: grupos de convivência. Sobre essa terminologia, Trentini et al. 13:70 conceitua como categoria que inclui vários subtipos de grupo quais sejam: grupos de ajuda-mútua, de apoio, de educação, de terapia e assim por diante. Eles tem o objetivo de crescimento pessoal. Na pesquisa, os grupos focais têm sido muito utilizados como técnica para obter dados, enquanto que os grupos de convergência têm a finalidade de desenvolver 0 crescimento pessoal e a pesquisa ${ }^{13}$.

\section{DIFERENTES ÂNGULOS PARA ABORDAR AS ATIVIDADES GRUPAIS}

As práticas grupais, para alguns autores da Enfermagem, como Alonso ${ }^{14}$ e Zampieri ${ }^{15}$, são consideradas num processo educativo em saúde, com ênfase na estruturação dos grupos e sua natureza interativa e de relação, respectivamente. Valorizando a concepção de educação em saúde em grupo, Alonso ${ }^{14}$ relata a necessidade de conhecer os conceitos sobre saúde, educação e os preceitos teóricos sobre grupo, bem como conhecer sua gênese, as fases de seu desenvolvimento, os papéis de seus membros e do líder, as possíveis formas de comunicação, facilitando a interação do profissional enfermeiro nessa prática.

Munari et al. ${ }^{16}$ são pioneiras da enfermagem na contribuição literária sobre grupos, com a publicação do primeiro livro específico sobre o assunto, intitulado Enfermagem e Grupos, no qual utilizam o respaldo de alguns estudiosos da Psicossomática. As práticas grupais são uma das formas de intervenção da educação em saúde muito utilizadas pela Enfermagem. Assim, esse tipo de abordagem é muito freqüentemente mencionada nos estudos de enfermeiros sobre estas práticas.

Trentini et al. ${ }^{13}$ trazem a discussão das práticas grupais como fonte tecnológica para a Enfermagem partindo do entendimento que a tecnologia é o saber - fazer intencional, mediado pela razão do ser humano e suas experiências ${ }^{17}$. A tecnologia é muito mencionada nos artigos escritos por enfermeiros. Merhy et al. ${ }^{18}$ trazem a concepção da tecnologia como um trabalho vivo em ato, sendo um processo que busca a produção de algo. 0 grupo como tecnologia pode ser visto como ato vivo que busca a produção, não de bens materiais, mas de uma determinada ação.

A abordagem dos grupos, como fonte tecnológica, traz a idéia da atividade grupal como produção. Ela é importante para a Enfermagem pois, no contexto hospitalar, as atividades educativas deste teor não são 
vistas com este enfoque. Entretanto, esta abordagem utilizada poderia ser utilizada como estratégia na discussão junto à gerência ou direção de instituições para implementação deste recurso à clientela.

0 tipo de abordagem que consideramos mais relevante e que traduz a importância desta atividade para o profissional enfermeiro é a de estratégia. Para a Enfermagem, esses grupos demonstram ser de grande valia, estando relacionados à estratégia para determinadas ações. Como exemplo, os estudos de Lucchese $^{19}$ e os de Côrrea, Souza e Saeki20 referem os grupos operativos como estratégia pedagógica em cursos de graduação em enfermagem. $E$ o estudo de Macie ${ }^{20}$ refere os grupos operativos como estratégia de socialização para idosos. Ainda se destaca Simões ${ }^{21}$, que apresenta um olhar estratégico desses grupos sobre a assistência de enfermagem, já vislumbrada antes nos estudos de Villella22 com mulheres em depressão.

0 termo estratégia é entendido como a arte de explorar condições favoráveis e aplicar os meios disponíveis ${ }^{4}$. Por isso, pensamos ser mais adequado aplicar este termo na abordagem do trabalho em grupo porque ele facilita a utilização do que se tem disponível numa instituição de saúde, sendo ele por si próprio a condição favorável.

A exemplo disto, no aspecto físico, a atividade grupal pode ser realizada numa enfermaria, numa sala de espera ou ao ar livre, na falta de uma sala fechada. No aspecto humano, a atividade não necessita exclusivamente de um único profissional para essa tarefa, podendo o mesmo estar na assistência, concomitantemente. E também é adequada, por economizar tempo atingindo um número maior de pessoas em menor quantidade de tempo. Essa visão de

\section{Referências}

1. Pichon-Riviére E. O Processo grupal. Tradução de Marco Aurélio Fernandez Velloso e revisão Mônica SM da Silva. $6^{a}$ ed. São Paulo (SP): Martins Fontes; 2000.

2. Mello Filho J. Psicossomática hoje. Porto Alegre (RS): Artes Médicas; 1992.

3. Zimerman DE. Classificação geral dos grupos. In: Zimerman DE et al. Como trabalhamos com grupos. Porto Alegre (RS): Artes Médicas; 1997.

4. Ferreira ABH. Novo Aurélio Século XXI: 0 dicionário da língua portuguesa. 3a ed. Revisada e ampliada. Rio de Janeiro (RI): Nova Fronteira; 1999. estratégia está relacionada com os objetivos traçados para essa atividade, podendo ser o trabalho de grupo uma estratégia de ensino-aprendizagem, socialização, autocuidado, humanização, entre outros.

\section{CONCLUSÃO}

A necessidade de trabalhos educativos para nossa população é identificada em todas as especialidades da área de saúde. Utilizar-se de meios que facilitem estes trabalhos, como os grupos por exemplo, é o que os profissionais enfermeiros precisam para promoverem a saúde a baixos custos e de forma eficaz.

As diferentes abordagens relativas ao trabalho de grupo na Enfermagem trazem novas perspectivas que reafirmam a importância dessa atividade em nossa profissão, principalmente quando ela adota a visão de estratégia.

A discussão em torno dessa temática é propícia por ser uma prática que a Enfermagem sempre utilizou. Por isso, faz-se necessária a aplicação de um conteúdo técnico-científico na estruturação dos grupos, para que bons resultados possam ser obtidos no exercício dessa atividade.

Por último, a visão Psicossomática ainda precisa ser melhor explorada nos estudos da Enfermagem, uma vez que ela se constitui no berço dos trabalhos grupais. Assim, ampliar os conhecimentos sobre as terminologias e tipos de abordagem, bem como os fundamentos teóricos que sustentam os trabalhos de grupo é condição sine qua non para que os grupos possam contribuir mais efetivamente para a qualidade do trabalho de enfermagem a ser desenvolvido junto à clientela.

5. Ficsmann JB. Como agem os grupos operativos? In: Zimerman DE et al. Como trabalhamos com grupos Porto Alegre (RS): Artes Médicas; 1997.

6. Saidon 0l. Grupo operativo de Pichon-Riviére: guia terminológico para construção de uma teoria crítica dos grupos operativos. In: Baremblitt G. Grupos: teorias e técnicas. 2ed. Rio de Janeiro (RJ): Graal; 1986. p.169-200.

7. Coronel LCl. Grupos de reflexão. In: Zimerman DE et al. Como trabalhamos com grupos. Porto Alegre (RS): Artes Médicas; 1997. p. 345-49.

8. Delors J. Educação: um tesouro a descobrir. Relatório para a Unesco da Comissão Internacional sobre a Educação para o SécuIo XXI. $5^{\text {a }}$ ed. Cortez; 2001. 
9. Barros CASM. Grupos de Auto-ajuda. In: Zimerman DE et al. Como trabalhamos com Grupos. Porto Alegre (RS): Artes Médicas; 1997. p. 107-17.

10. Mello Filho J. Grupoterapia com pacientes somáticos: 25 anos de experiência. In: Zimerman DE et al. Como trabalhamos com Grupos. Porto Alegre: Artes Médicas; 1997.p.185-204.

11. Mello Filho J. Grupos no Hospital Geral: ambulatório, enfermaria e serviços especializados. In: Mello Filho J. et al. Grupo e corpo: psicoterapia de grupo com pacientes somáticos. Por to Alegre (RS): Artes Médicas Sul; 2000. p.131-43.

12. Chazan LF. Grupos homogêneos interdisciplinares. In: Mello Filho J et al. Grupo e corpo: psicoterapia de grupo com pacientes somáticos. Porto Alegre (RS): Artes Médicas Sul; 2000. p.163-72.

13. Trentini M, Gonçalves LHT. Pequenos grupos de convergência: um método no desenvolvimento de tecnologias na enfermagem. Texto $\&$ Contexto Enferm 2000 jan/abr; 9(1): 63-78.

14. Alonso ILK. O Processo educativo em saúde na dimensão grupal. Texto \& Contexto Enferm 1999 jan/abr; 8 (1): 203-21.

15. Zampieri MFM. Vivenciando o processo educativo em enfermagem com gestantes de alto risco e seus acompanhantes. Texto $\&$ Contexto Enferm 1999 jan/abr ; 8(1): 203-21.

16. Munari DB, Rodrigues ARF. Enfermagem e grupos. Goiânia (GO): $A B ; 1997$.

17. Meier MJ, Cianciarullo TI. Tecnologia: um conceito em construção para o trabalhador em saúde. Texto \& Contexto Enferm 2002 jan/abr; 11(1): 31-49.

18. Merhy EE, Onocko R. Praxis en salud: un desafio para lo público. Buenos Aires (AR): Lugar Editorial; 1997.

19. Lucchese R. Grupo operativo como estratégia pedagógica em um curso de graduação em enfermagem: um espaço continente das vivências dos alunos quartanistas. [Dissertação de mestrado] São Paulo (SP): Escola de Enfermagem/ USP; 2000.

20. Côrrea AC, Souza MCB, Saeki T. Transição para o exercício profissional em enfermagem: uma experiência em grupo operativo. Esc Anna Nery Rev Enferm 2005 dez; 9(3): 421-28.

21. Maciel PM. A mulher idosa num grupo de convivência: um estudo na perspectiva da enfermagem. [tese de doutorado]. Rio de Janeiro (RJ): Escola de Enfermagem Anna Nery / UFRJ; 2002.

22. Simões FV. Grupos operativos: Uma estratégia para a assistência de enfermagem em uma unidade hospitalar de referência em câncer de mama. [dissertação de mestrado]. Rio de Janeiro (RJ): Escola de Enfermagem Anna Nery/ UFR); 2004.

23. Villela DVAL. 0 grupo operativo como estratégia para a assistência de enfermagem: mulheres em depressão. [dissertação de mestrado] São Paulo: Escola de Enfermagem/USP; 2000.

\section{Notas}

a 0 eixo norteador deste trabalho foi a dissertação de mestrado defendida em 2004, na Escola de Enfermagem Anna Nery(EEAN) / Universidade Federal do Rio de Janeiro (UFRJ), intitulada "Grupos operativos: uma estratégia para a assistência de enfermagem em uma unidade hospitalar de referência em câncer de mama".

\section{Sobre as Autoras}

\section{Fabiana Verdan Simöes}

Membro da Comissão de Ensino de Enfermagem do Instituto Nacional do Câncer (INCA). Enfermeira especializada em Enfermagem Oncológica pelo INCA - Escola de Enfermagem Anna Nery (EEAN) / UFRJ. Mestre em Enfermagem pela EEAN / UFRJ. Email: fverdan@ hotmail.com.

\section{Marluci Andrade Conceição Stipp}

Doutora em Enfermagem e Pesquisadora do Núcleo de Pesquisa em Gerência, Exercício Profissional e Educação em Enfermagem e Professora Adjunta do Departamento de Metodologia da EEAN / UFRJ. 